\title{
A Finite Horizon Production Model with Variable Production Rates and Constant Demand Rate
}

\author{
ZVI GOLDSTEIN $\dagger$ \\ zgoldstein@fullerton.edu \\ Department of Information Systems and Decision Science, College of Business and \\ Economics, California State University, Fullerton, CA 92834, USA
}

\begin{abstract}
In this paper we present a finite horizon single product single machine production problem. Demand rate and all the cost patterns do not change over time. However, end of horizon effects may require production rate adjustments at the beginning of each cycle. It is found that no such adjustments are required. The machine should be operated either at minimum speed (i.e. production rate $=$ demand rate; shortage is not allowed), avoiding the buildup of any inventory, or at maximum speed, building up maximum inventories that are controlled by the optimal production lot size.
\end{abstract}

Keywords: Supply Chain, Production, Finite Horizon

\section{Introduction}

In this paper we present a single product single machine finite horizon production problem. The production rates can be adjusted at the beginning of each production run. When the planning horizon is infinitely long, stationary policies are optimal if the cost patterns and the demand rate do not change over time. The classic Production Lot Size model (with a fixed production rate) is one example where the production lot size is constant.

Production rates, however, can be adjusted by changing the production speed and/or by short pauses between consecutive items when feeding the machine. This can be more economical than letting the machine work at a constant production rate (see discussion in Silver (1990)). For other studies which allow a changing production rate in each cycle see Inman and Jones (1989), Gallego (1993). They build on the observation that stationary policies are optimal (all cycles are the same). From this standpoint, the finite horizon case studied in this paper is different, since end of horizon

$\dagger$ Requests for reprints should be sent to Zvi Goldstein, Department of Information Systems and Decision Science, College of Business and Economics, California State University, Fullerton, CA 92834, USA. 
effects may lead to a changeable production rate even if the cost profile remains the same throughout the time-horizon.

A finite horizon model fits cases where production is discontinued at some predictable time in the future. One reason for discontinuing a production line is the introduction of new competing products, which render the old product obsolete (a common phenomenon in the PC industry for example). Occasionally, even though an infinite horizon model is justifiable, a rolling horizon approach is implemented where a series of finite horizon problems is used as a practical approach to production planning. Applying the forecast horizon theory to production control also calls for the repeated solution of finite horizon problems, in order to identify the optimal first decision of the infinite horizon problem (See, for example, Bean et al (1990); Goldstein and Mehrez (1996)).

This paper is organized as follows. In section 2 the model is presented, and in section 3 an analysis leading to the characteristics of the optimal production strategy is presented. In section 4 we provide an example that illustrates the solution procedure. We summarize the results in section 5.

\section{The Model}

Consider a single product manufactured on a single machine. The machine production rate can be controlled, by adjusting its speed. Time-dependent cost associated directly with the production process (i.e. wages) can be reduced when the total production time is shortened. This, in turn, can be achieved by operating the machine at a higher speed. However, running the machine faster may create large inventories, thus increasing the holding cost. The total inventory cost is controlled by the proper selection of the lot size.

It is clear that both the production lot size and the production rate affect the total cost. We allow the production rate to be adjusted at the beginning of each production run. This adjustment may be necessary in order to minimize the total cost under the finite horizon condition of our model. It is assumed that the demand rate is constant. When the time horizon is not too long, this assumption should lead to useful results even for the case where the actual demand pattern varies over time. In formulating the finite horizon production model we use the following definitions: 


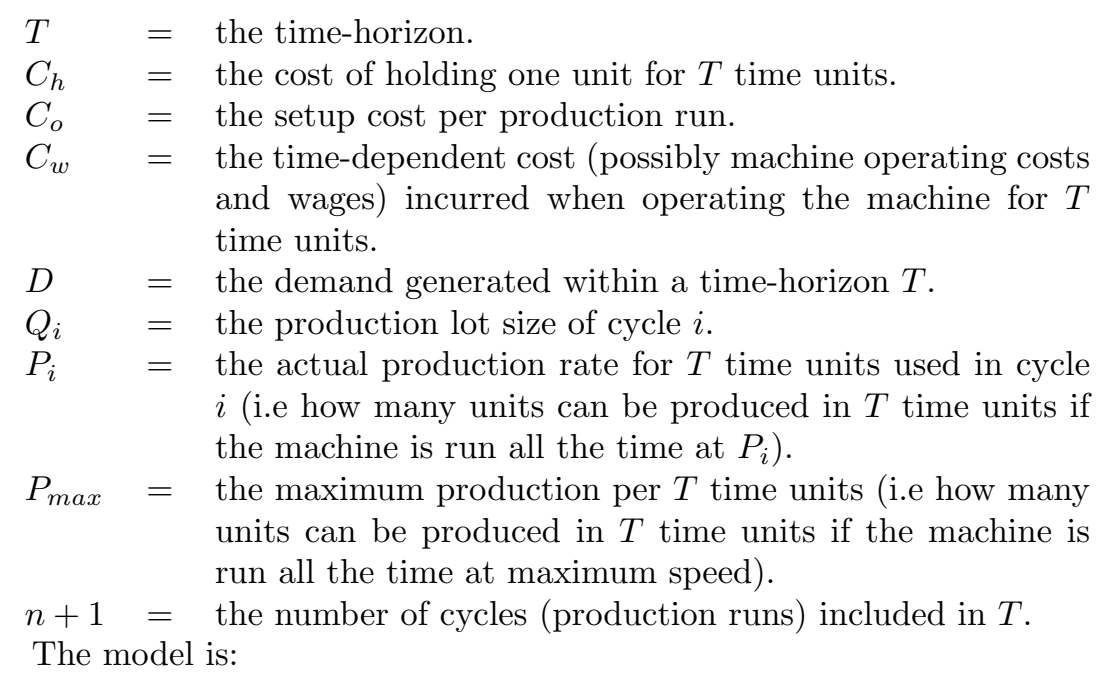

$$
\begin{array}{ll}
\min & T C=(n+1) C_{o}+\sum_{i=1}^{n+1}\left[C_{h}\left(1-\frac{D}{P_{i}}\right)\left(\frac{Q_{i}^{2}}{2 D}\right)+C_{w} \frac{Q_{i}}{P_{i}}\right] \\
\text { s.t. } & \\
& \sum_{i=1}^{n+1} Q_{i}=D \\
& P_{i} \geq D \text { and } P_{i} \leq P_{\max }
\end{array}
$$

Constraint (2) guarantees that all the demand generated within time $T$ is met, while the first constraint in (3) assures that the production system will not be out-of-stock during any cycle. The second constraint of (3) limits the speed of the machine due to technological limitations and/or managerial decisions.

\section{Analysis}

We study several characteristics of the optimal solution. First, we show that only two possible schedules can be optimal: either the production rate stays during the time horizon at the minimum speed $\left(P_{i}=D\right)$, or the production rate is at its maximum speed possible $\left(P_{i}=P_{\max }\right)$, which we call a boundary-type solution. 
THEOREM 1 For any given cycle $i$, it is optimal to run the machine either at a maximum production rate $P_{i}=P_{\max }$, or at a minimum production rate $P_{i}=D$

Proof: Rearranging the total cost function (1) for the optimal set of $Q_{i}$ 's:

$$
\left.\left.T C=(n+1) C_{o}+\sum_{i=1}^{n+1}\left[C_{w} Q_{i}-C_{h} \frac{Q_{i}^{2}}{2}\right)\right]\left(\frac{1}{P_{i}}\right)+C_{h} \frac{Q_{i}^{2}}{2 D}\right\} .
$$

This is a monotone function in each individual $P_{i}$. For any given $Q_{i}$, if $C_{w}-C_{h} \frac{Q_{i}}{2}>0$, the function is monotonically decreasing in $P_{i}$, in which case $P_{i}=P_{\max }$. If $C_{w}-C_{h} \frac{Q_{i}}{2}<0$ the function is monotonically increasing in $P_{i}$ in which case $P_{i}=D$.

Note that when $C_{w}-C_{h} \frac{Q_{i}}{2}=0$ any production rate is equally attractive. In this case a boundary solution is also optimal.

LEMma 1 The order of the cycles does not affect the total cost.

Proof: The order is irrelevant to equation (1).

Lemma 2 It is non-optimal to run the machine at $P_{i}=D$ in more than one cycle.

Proof: Two cycles with production rate $P=D$ are better placed in a row (change of order is allowed by Lemma 1 ) and merged into one cycle rather than be separated because there is no inventory and one set-up cost is saved. The argument extends to any number of cycles.

Theorem 1 and Lemma 2 yield three possible production schedules that must be considered when looking for the optimal solution.

$S_{\text {min }}$ - the machine operates at the smallest rate possible $P_{i}=D$ throughout the time horizon.

$S_{\max }$ - the production rate in each cycle is $P_{i}=P_{\max }$

$S_{\text {mix }}-n>0$ cycles with $P_{i}=P_{\max }$, and one cycle with production rate of $P_{i}=D$.

\subsection{The Total Cost for $S_{\min }$}

Substituting in Equation (1) $P_{i}=Q_{i}=D$ and $n=0$ yields:

$$
T C=C_{o}+C_{w}
$$




\subsection{The Total Cost for $S_{\max }$}

For a given $n, Q_{i}=\frac{D}{n+1}$

$$
T C=(n+1) C_{o}+C_{h}\left(1-\frac{D}{P_{\max }}\right) \frac{D}{2(n+1)}+C_{w} \frac{D}{P_{\max }}
$$

If we treat $n$ as a continuous variable, the optimal $n+1$ is $\sqrt{\frac{C_{h}\left(1-\frac{D}{P}\right) D}{2 C_{o}}}$. Since $T C$ is convex in $n$, the number of runs $n_{\max }(=n+1)$ is calculated as follows:

1. Calculate $n^{*}=\sqrt{\frac{C_{h}\left(1-\frac{D}{P}\right) D}{2 C_{o}}}$.

2. Define $n^{-}$as the integer immediately below $n^{*}$, and $n^{+}$as the integer immediately above $n^{*}$.

3. If $T C\left(n^{-}\right)<T C\left(n^{+}\right)$then $n_{\max }=n^{-}$, else $n_{\max }=n^{+}$.

\subsection{The case $S_{m i x}$}

Theorem 2 For any two cycles $i$ and $j$, if $P_{i}=P_{j}$, then $Q_{i}=Q_{j}$.

Proof: Let $Q$ be the total production in cycles $i$ and $j$ combined. Let $P_{i}=P_{j}=P$. The total cost for the two cycles is

$C=2 C_{o}+C_{h}(1-D / P)\left(Q_{i}^{2} / D\right)+C_{h}(1-D / P)\left(Q-Q_{i}\right)^{2} D+C_{w}(Q / P)$.

The optimal order for these cycles must satisfy $d C / d Q_{i}=0$ which results in $Q_{i}=Q / 2$.

LEMma 3 For any given cycle the optimal production rate is $P_{i}=P_{\max }$ if

$$
Q_{i} \leq \frac{2 C_{w}}{C_{h}}
$$

Otherwise, the optimal production rate is $P_{i}=D$.

Proof: By the proof of Theorem 1: If $C_{w}-C_{h}\left(D_{i} / 2\right)>0$ it is optimal to run the machine at $P_{i}=P_{\max }$.

In Theorem 3 we show that $S_{m i x}$ is non-optimal.

THEOREM 3 Let $n$ be the number of production runs scheduled at a production rate of $P=P_{\max }$. Then, at least one of the following production schedules is optimal: 
$S_{\min }: n=0 ; P=D$. The machine operates continuously at the minimum production rate.

$S_{\max }: n_{\max }$ cycles with production lot size of $Q=D / n_{\max }$ units each at maximum speed $\left(P=P_{\max }\right)$.

Proof: We show that $S_{\text {min }}$ is a better schedule than $S_{m i x}$ thus, schedule $S_{m i x}$ is non-optimal. For the schedule $S_{m i x}$ there are $n>0$ cycles with $P=P_{\max }$ and one cycle with $P=D$. If $n=0$, it is schedule $S_{\min }$. The total cost is

$$
T C=(n+1) C_{o}+n\left[C_{h}\left(1-\frac{D}{P_{\max }}\right)\left(\frac{q^{2}}{2 D}\right)+C_{W} \frac{q}{P_{\max }}\right]+C_{W} \frac{D-n q}{D}
$$

where $Q_{i}=q$ for all $i=1,2, \ldots, n$ by Theorem 2 .

To solve (6) we need to find the optimal production lot $q^{*}$ common to $n$ cycles where $P=P_{\max }$. From (6), for any given $n>0, T C(q)$ is convex in $q$ so $q^{*}$ can be found by

$$
\begin{aligned}
\frac{d T C}{d q} & =n\left[C_{h}\left(1-\frac{D}{P_{\max }}\right) \frac{q}{D}+\frac{C_{w}}{P_{\max }}\right]-n \frac{C_{w}}{D} \\
& =\frac{n}{D}\left(1-\frac{D}{P_{\max }}\right)\left(C_{h} q-C_{w}\right)=0
\end{aligned}
$$

Solving for $q^{*}$ we get

$$
q^{*}=\frac{C_{w}}{C_{h}}
$$

since $n>0$ and $D<P_{\max }$. This solution can be optimal for $S_{\operatorname{mix}}$ only if it is feasible. It is infeasible when $C_{w} / C_{h}>D$, in which case we need to modify $q^{*}$ and determine that, $q^{*}=D$ since $T C$ is convex in $q$. This solution is one production run at maximum speed, plus one unneeded setup for the empty "cycle" run at $P=D$. This solution is inferior to $S_{\max }$ because it has an extra set-up cost. The solution (7) is feasible thus optimal for $S_{m i x}$ if $C_{w} / C_{h} \leq D$. The total cost $T C$ for the optimal $S_{m i x}$, is calculated by (6) when substituting $q=C_{w} / C_{h}$ :

$$
\begin{aligned}
T C\left(S_{\text {mix }}\right)= & (n+1) C_{o}+n\left[C_{h}\left(1-\frac{D}{P_{\max }}\right)\left(\frac{\left(\frac{C_{w}}{C_{h}}\right)^{2}}{2 D}\right)+C_{W}\left(\frac{\frac{C_{w}}{C_{h}}}{P_{\max }}\right)\right] \\
& +C_{W} \frac{D-n \frac{C_{w}}{C_{h}}}{D} \\
= & C_{o}+C_{w}+n\left[C_{o}-\frac{1}{2 D}\left(1-\frac{D}{P_{\max }}\right)\right] \frac{C_{w}^{2}}{C_{h}} .
\end{aligned}
$$


If $\left[C_{o}-\frac{1}{2 D}\left(1-\frac{D}{P_{\max }}\right)\right] \frac{C_{w}^{2}}{C_{h}} \geq 0$, then $T C\left(S_{m i x}\right) \geq C_{o}+C_{w}$ for all $n \geq 1$ and therefore $S_{\min }$ is at least as good as $S_{m i x}$. If $\left[C_{o}-\frac{1}{2 D}\left(1-\frac{D}{P_{\max }}\right)\right] \frac{C_{w}^{2}}{C_{h}}<$ 0 , then $T C\left(S_{\text {mix }}\right)$ is minimized at the maximum feasible value for $n$ (defined as $\left.n_{m i x}\right)$. That is, $n_{m i x}$ is the largest integer that satisfies $n\left(C_{w} / C h\right)<D$.

Applying $n_{m i x}$ to the schedule $S_{m i x}$ leaves less than $C_{w} / C_{h}$ units of demand to be covered by the cycle where $P=D$. By Lemma 3 this cannot be an optimal schedule. $\square$.

Following Theorem 3 only $S_{\text {min }}$ (running the machine at minimum production rate all the time), and $S_{\max }$ (running the machine for $n_{\max }$ cycles at maximum production rate) can be optimal. the total cost need to be calculated for each case, and the better one selected as the optimal solution.

\section{Example}

Find the optimal production schedule for the following production problem:

$C_{o}=\$ 1000$ per setup, $C_{h}=\$ 20$ per unit per time horizon, $C_{w}=\$ 25,000$ per time horizon, $D=5000$ units, $P_{\max }=8000$ units per time horizon.

\subsection{Solution}

Since $2 C_{w} / C_{h}=25000 / 20=2500<D=5000$, both $S_{\max }$ and $S_{\min }$ should be considered. Let us first find the best production plan under $S_{\max }$. To calculate $n_{\max }$ find $n^{*}=\left\{\left[C_{h}(1-D / P) D\right] /\left[2 C_{o}\right]\right\}^{1 / 2}=4.33$. Thus, $n^{--}=4$ and $n^{+}=5$. Using equation (6) $T C(n=4)=24312.50$, and $T C(n=5)=24,859.38$, so $n_{\max }=4$ and $T C\left(S_{\max }\right)=24312.50$. Since $T C\left(S_{\min }\right)=C_{o}+C_{w}=26,000$, the optimal solution is to schedule 4 production runs of $Q=5000 / 4=1250$ at $P=8000$ units each. The machine will be busy $Q / P=1250 / 8000=15.625 \%$ of the time.

\section{Summary}

In this paper we formulate a finite horizon production problem of a single product on a single machine, where production rates can be adjusted at the beginning of each cycle. Although demand rate is considered constant, and the cost profile does not change over time, the end of horizon effect may call for different production rates. It is found that only two possible schedules can be optimal. The first schedule $S_{\text {min }}$, calls for running the machine all the time at minimum speed $P_{\text {min }}=D$ (where $P_{\text {min }}$ represents the smallest 
production rate allowed in order to prevent shortages). No inventories are built and the machine works constantly. The second schedule $S_{\max }$ calls for running the machine at maximum speed in each production cycle for short production runs.

Natural extensions of the model presented in this paper are the multiproduct case, and the variable-demand rate case, currently investigated by the author.

\section{References}

1. Bean, J.C., R.L Smith., and J.B. Lasserre, (1990). "Denumerable State Nonhomogeneous Markov Decision Processes." J. of Math. Anal. And Appl., 153, 64 77 .

2. Gallego, G. (1993). "Reduced Production Rates in the Economic Lot Scheduling Problem." International Journal of Production Research, 31(5), 1035 - 1046.

3. Goldstein, Z., and A. Mehrez (1996). "Replacement of Technology when a New Technological Breakthrough is Ecpected", Eng. Optimization, 27, 265 - 278.

4. Inman and Jones (1989). "When is the Economic Lot Scheduling Problem Easy?" HE Transactions, 21, 11 - 20 .

5. Khouja, M. (1999). "The Economic Lot and Delivery Scheduling Problem: Common Cycle, Rework and Variable Production Rate Case." HE Transactions, forthcoming.

6. Moon, 1., G. Gallego, and D. Simchi-Levi (1991). "Controllable Production Rates in a Family Production Context." International Journal of Production Research, $29,2459-2470$.

7. Silver, E. A. (1990). "Deliberately Slowing Down Output in a Family Production Context." International Journal of Production Research, 28, (1), 17-27. 


\section{Mathematical Problems in Engineering}

\section{Special Issue on}

\section{Modeling Experimental Nonlinear Dynamics and Chaotic Scenarios}

\section{Call for Papers}

Thinking about nonlinearity in engineering areas, up to the 70 s, was focused on intentionally built nonlinear parts in order to improve the operational characteristics of a device or system. Keying, saturation, hysteretic phenomena, and dead zones were added to existing devices increasing their behavior diversity and precision. In this context, an intrinsic nonlinearity was treated just as a linear approximation, around equilibrium points.

Inspired on the rediscovering of the richness of nonlinear and chaotic phenomena, engineers started using analytical tools from "Qualitative Theory of Differential Equations," allowing more precise analysis and synthesis, in order to produce new vital products and services. Bifurcation theory, dynamical systems and chaos started to be part of the mandatory set of tools for design engineers.

This proposed special edition of the Mathematical Problems in Engineering aims to provide a picture of the importance of the bifurcation theory, relating it with nonlinear and chaotic dynamics for natural and engineered systems. Ideas of how this dynamics can be captured through precisely tailored real and numerical experiments and understanding by the combination of specific tools that associate dynamical system theory and geometric tools in a very clever, sophisticated, and at the same time simple and unique analytical environment are the subject of this issue, allowing new methods to design high-precision devices and equipment.

Authors should follow the Mathematical Problems in Engineering manuscript format described at http://www .hindawi.com/journals/mpe/. Prospective authors should submit an electronic copy of their complete manuscript through the journal Manuscript Tracking System at http:// mts.hindawi.com/ according to the following timetable:

\begin{tabular}{|l|l|}
\hline Manuscript Due & December 1,2008 \\
\hline First Round of Reviews & March 1, 2009 \\
\hline Publication Date & June 1,2009 \\
\hline
\end{tabular}

\section{Guest Editors}

José Roberto Castilho Piqueira, Telecommunication and Control Engineering Department, Polytechnic School, The University of São Paulo, 05508-970 São Paulo, Brazil; piqueira@lac.usp.br

Elbert E. Neher Macau, Laboratório Associado de Matemática Aplicada e Computação (LAC), Instituto Nacional de Pesquisas Espaciais (INPE), São Josè dos Campos, 12227-010 São Paulo, Brazil ; elbert@lac.inpe.br

Celso Grebogi, Center for Applied Dynamics Research, King's College, University of Aberdeen, Aberdeen AB24 3UE, UK; grebogi@abdn.ac.uk 